\title{
Sulla struttura dello spazio-tempo $\left(^{*}\right)(* *)$.
}

\author{
Renato Grassini (Napoli)
}

Summary. - The paper fits in the sphere of foundational researches on Physical Geometry. A methodological line is followed which aims at giving general mathematical models of space and time and capturing the foundational value of the corresponding chronogeometrical structures; along this line a canonical model of space-time is provided which forms the largest substratum underlying both the Newtonian and the relativistic models of space-time, whose peculiar features are then derived by means of the invariance conditions imposed upon the chronogeometrical structures by some additional physical hypotheses.

\section{Introduxione.}

Con lo sviluppo della Teoria della Relatività è invalso il punto di vista di considerare elemento primitivo della Geometria Fisica, sia nelle teorie newtoniane che in quelle relativistiche, lo spazio-tempo o universo degli eventi, di cui poi ciascuna teoria è intesa fornire un particolare modello matematico. Ne è seguita quindi l'esigenza di analizzare in dettaglio le strutture inerenti i suddetti modelli e specialmente le relazioni fra tali strutture e le assunzioni fisiche che esse esprimono [1].

Per tal via si è venuto costituendo un quadro di ricerche comparate sui fondamenti della Geometria Fisica newtoniana e relativistica, nel quale si distinguono due linee direttrici principali: da un lato le ricerche sugli aspetti relativistici dello spaziotempo newtoniano [2-5], rivolte ad analizzare in questo le successive strutture geometrico-differenziali (conforme, proiettiva, affine, metrica) assunte come fondamentali in Relatività $[6,7]$; dall'altro le ricerche sugli aspetti newtoniani dello spazio-tempo relativistico [8-12], rivolte a derivare in questo strutture corrispondenti alle nozioni newtoniane di spazio e tempo ed aventi il significato operazionale di osservabili fisiche.

Recentemente si è venuta poi configurando una terza linea di ricerca, rivolta alla descrizione formale generale dello spazio e del tempo ed al recupero del valore fondazionale delle corrispondenti strutture cronogeometriche.

Precisamente, nell'ambito di tale linea di ricerca, C. Harrison ha introdotto [13] nell'universo degli eventi strutture cronogeometriche globali (sistema spazio-temporale, classe canonica di sistemi spazio-temporali) che risultano un comune fondamento delle teorie piatte, newtoniane e relativistiche, dello spazio-tempo.

(*) Lavoro eseguito nell'ambito del Gruppo Nazionale per la Fisica Matematica del C.N.R.

(**) Entrata in Redazione il 5 dicembre 1978. 
D'altra parte, nell'ambito della medesima linea di ricerca, ho introdotto [14-16] in un modello generale d'universo, costituito da una 4-varietà a connessione affine [17], strutture cronogeometriche locali (1-forma temporale, metrica spaziale, connessione spaziale) che risultano comuni anche alle teorie non piatte dello spazio-tempo.

$\mathrm{Nel}$ presente lavoro, sulla base di quest'ultima impostazione metodologica, propongo una generalizzazione della teoria formale di C. Harrison che, sintetizzando i risultati cronogeometrici dei vari lavori precedentemente citati, realizza un approccio fondazionale unificato a tutte le teorie, newtoniane e relativistiche, dello spazio-tempo. Momento centrale di tale approccio è l'individuazione di un modello canonico di spaziotempo, che evidenzia il completo bagaglio di strutture cronogeometriche e corrispondenti assunzioni fisiche costituenti il comune fondamento delle suddette teorie.

Il piano del lavoro $\left(^{1}\right)$ è il seguente:

(i) In una 4-varietà $M$, assunta come modello matematico fondamentale d'universo, si ridefiniscono $(\$ \S 1,2)$ le strutture cronogeometriche di C. Harrison, in corrispondenza a nozioni locali di spazio e tempo intese nella molteplicità delle relative determinazoni fisiche.

(ii) L'introduzione in $M$ di una classe canonica $C$ di sistemi spazio-temporali e di una connessione affine $D$, collegate da naturali condizioni di compatibilità, conduce $(\S 3)$ al preannunciato modello canonico di spazio-tempo.

(iii) Condizioni di invarianza imposte all'interno di $C$ da un principio di isotropia ottica e dal suo limite newtoniano conducono $(\$ \S 4,5)$ alle specializzazioni einsteiniana e newtoniana del modello canonico. La prima viene a identificarsi (nel caso generale) con lo spazio-tempo di Einstein-Cartan [23, 24], di cui poi la seconda fornisce l'analogo newtoniano.

\section{1. - Sistema spazio-temporale.}

\subsection{Definizione.}

Sia $M$ una varietà differenziale reale 4-dimensionale, assunta come modello matematico fondamentale d'universo.

Le nozioni di spazio e tempo (e quelle correlate di misura ed evoluzione) saranno modellate in $M$ mediante la struttura di sistema spazio-temporale $S$, definito come segue:

1-1 Defrnzione. - $S$ è un sistema spazio-temporale in $M$ se è

$$
S=(I I, g, \nabla)
$$

(1) Per le tecniche matematiche in esso adoperate (teoria dei fibrati vettoriali e strutture associate), cf. [18-20]; per le tecniche logiche (metodo di assiomatizzazione mediante predicati teorico-insiemistici), ef. $[21,22]$. 
denotando $(I, g, \nabla)$ un insieme costituito da una struttura quasi-prodotto $I$, una metrica lorentziana $g$ ed una connessione affine $\nabla$ verificanti i seguenti assiomi:

(S1) $\Pi$ decompone il fibrato tangente $T M$ nella somma di Whitney

$$
T M=\sigma \oplus \theta
$$

dove $\sigma$ denota una distribuzione 3-dimensionale e $\theta$ una distribuzione orientata 1-dimensionale su $M$.

(S2) $g$ si decompone nella somma $\left({ }^{2}\right)$

$$
g=g_{\sigma} \circ \mathfrak{T}_{\sigma}+g_{\theta} \circ \mathfrak{T}_{\theta},
$$

dove $g_{\sigma}$ denota una metrica fibrata definita positiva in $\sigma$ e $g_{\theta}$ una metrica fibrata definita negativa in $\theta$.

(S3) $\nabla$ si decompone nella somma

$$
\nabla=\nabla_{\sigma} \circ \mathfrak{S}_{\sigma}+\nabla_{\theta} \circ \mathfrak{I}_{\theta}
$$

dove $\nabla_{\sigma}$ denota una connessione metrica in $\sigma$ e $\nabla_{\theta}$ una connessione metrica in $\theta$.

\subsection{Proprietà.}

a) L'assioma (S1) descrive, precisandone la struttura topologica, le nozioni di spazio e tempo locali.

Precisamente, $\Pi$ definisce nello spazio tangente $T_{x} M$ (per ogni $x \in M$ ) due relazioni di equivalenza, caratterizzate da quozienti $\Sigma_{x}$ e $\Theta_{x} \operatorname{costituiti~dai~laterali~di~} \theta_{x}$ e $\sigma_{x}$ rispettivamente, tali che, stante (1.1), $T_{x} M$ risulta isomorfo, tramite le corrispondenti proiezioni canoniche, al prodotto cartesiano $3+1$-dimensionale $\Sigma_{x} \times \Theta_{x}$.

Identifieando $T_{x} M$ con un intorno infinitesimo di $x$ in $M$, le suddette relazioni saranno allora interpretate come relazioni di genidentità e simultaneità locali in $M$, ossia relazioni i cui quozienti $\Sigma_{x}$ e $\Theta_{x}$ (isomorfi a $\sigma_{x}$ e $\theta_{x}$ rispettivamente) definiscono spazio e tempo locali in $M$ (per questi assumendo valida l'ordinaria geometria affine). Il prefissato orientamento su $\theta$, che sarà denotato con $f$, corrisponderà poi all'assunzione che sia univocamente determinato il verso del tempo.

Con riferimento al sistema $S, \sigma$ e $\theta$ si diranno distribuzioni spaziale e temporale e II, che le sintetizza, struttura spazio-temporale.

Si osservi che la distribuzione temporale $\theta$, essendo 1-dimensionale, è senz'altro integrabile. È pertanto possibile riguardare le varie relazioni di genidentità locale in $T M$.

( ${ }^{2}$ Con $\mathfrak{f}_{\sigma}: T M \rightarrow \sigma, \mathfrak{f}_{\theta}: T M \rightarrow \theta$ si indicheranno le proiezioni su $\sigma$ e $\theta$ definite da $\Pi$ 
come determinazioni di un'unica relazione di genidentità globale, caratterizzata da un quoziente $\Sigma$ costituito dalle sottovarietà integrali massimali di $\theta$. Lo spazio globale $\Sigma$ (recante generalmente una struttura differenziale, ma non affine) sarà inteso rappresentativo di un continuo 3 -dimensionale di particelle (reali o ideali) ovvero di un sistema di riferimento, costituente il supporto fisico di $\$$.

b) L'assioma (\$2) definisce la struttura metrica dello spazio e del tempo locali.

Precisamente, $g$ si decompone, stante (1.2), in una coppia di metriche degeneri (semidefinite e di opposte segnature), $g_{\sigma} \circ \mathfrak{T}_{\sigma}$ e $g_{\theta} \circ \mathfrak{S}_{\theta}$, che risultano invarianti per trasformazioni di $T M$ preservanti rispettivamente la genidentità e la simultaneità.

I relativi intervalli elementari saranno allora interpretati come intervalli spaziale e temporale, corrispondenti alle misure locali di distanza e durata (per queste assumendo valida l'ordinaria geometria euclidea).

Con riferimento al sistema $S, g_{\sigma}$ e $g_{\theta}$ si diranno metriche spaziale e temporale e $g$, che le sintetizza, metrica spazio-temporale $\left({ }^{3}\right)$.

Al fine di un'ulteriore caratterizzazione delle metriche $g$ verificanti l'assioma $(S 2)$, consideriamo, insieme alla classe $\mathfrak{L}$ delle metriche suddette, la classe $\mathfrak{L}^{\prime}$ delle coppie $(\tau, h)$ costituite da

(i) una 1-forma temporale $\tau\left({ }^{4}\right)$, ossia una 1-forma ovunque non nulla in $M$ verificante le condizioni

$$
\begin{gathered}
\operatorname{Ker}(\tau)=\sigma \\
\tau-\operatorname{or}(\theta)=f\left({ }^{5}\right)
\end{gathered}
$$

(una siffatta 1-forma, oltre a descrivere la struttura topologica e l'orientamento del tempo locale, definisce una metrica temporale $k$ data da $k \circ \mathfrak{T}_{\theta}=-\tau \otimes \tau$ );

(ii) una metrica spaziale $h\left({ }^{6}\right)$.

Ogni metrica $g \in \mathcal{L}$ è suscettibile, in modo univoco, della decomposizione

$$
g=-\tau \otimes \tau+h \circ \mathfrak{I}_{\sigma},
$$

in cui la coppia $(\tau, h) \in \mathfrak{L}^{\prime}$ è data da

$$
\begin{aligned}
\tau & =-g(\gamma) \\
h & =g_{\sigma},
\end{aligned}
$$

${ }^{(3)}$ L'introduzione in $M$ della metrica spazio-temporale $g$ consente di fornire un'interpretazione potenzialmente operativa alla struttura differenziale di $M$, che viene infatti ad am. mettere un atlante di coordinate interpretabili, nell'intorno infinitesimo di ciascun punto, come misure di distanza e durata.

(4) Cf. [14], pp. 721-722.

(5) Si è posto $\operatorname{Ker}(\tau)=\{u \in T M:\langle\tau, u\rangle=0\}$; inoltre $\tau$-or $(\theta)$ denota il $\tau$-orientamento di $\theta$ (definito dal generatore globale $\bar{\gamma}$ caratterizzato dalla condizione $\langle\tau, \bar{\gamma}\rangle=1$ ).

(6) Cf. $[16]$, p. 510 . 
denotando $\gamma$ il generatore globale $f$-orientato di $\theta$ caratterizzato dalla condizione

$$
g(\gamma, \gamma)=-1
$$

e $g(\gamma)$ la relativa immagine nell'isomorfismo definito da $g$ fra $T M$ e il suo duale.

D'aitra parte, ad ogni coppia $(\tau, h) \in \mathfrak{L}^{\prime}$ l'equazione $(1.6)$ associa evidentemente una metrica $g \in \mathfrak{L}$, sicchè sussiste la seguente proposizione:

1-2 Propostzione. - L'equazione (1.6) definisce una biiezione di $\mathfrak{L}$ su $\mathfrak{L}^{\prime}$.

e) L'assioma (S3) introduce una connessione tra le varie determinazioni dello spazio e del tempo locali.

Precisamente, $\nabla$ si decompone, stante (1.3), in una coppia di connessioni metriche in $M, \nabla_{\sigma} \circ \mathfrak{T}_{\sigma}$ e $\nabla_{\theta} \circ \mathfrak{S}_{\theta}$, che risultano invarianti per trasformazioni preservanti rispettivamente la genidentità e la simultaneità.

Le corrispondenti derivazioni covarianti saranno allora interpretate come derivazioni spaziale e temporale, atte a descrivere localmente (in accordo con l'ordinaria geometria euclidea) l'evoluzione relativa, spaziale e temporale, di grandezze fisiche.

Con riferimento al sistema $S, \nabla_{\sigma}$ e $\nabla_{\theta}$ si diranno connessioni spaziale $\left(^{7}\right)$ e temporale e $\nabla$, che le sintetizza, connessione spazio-temporale.

Al fine di un'ulteriore caratterizzazione della connessione spazio-temporale $\nabla$, consideriamone i coefficienti $\tilde{\omega}_{j}^{i}\left(^{8}\right)$ relativi alla generica sezione $s$ del fibrato

$$
B(\Pi)=\left\{\left(e_{i}\right) / e_{\alpha} \in \sigma, e_{0} \in \theta\right\}
$$

costituito dalle basi di $T M$ adattate a $\Pi$.

La decomponibilità (1.3), che equivale alla duplice condizione di $\sigma$ e $\theta$-riducibilità di $\nabla$, è espressa in $s$ dalle equazioni $\left({ }^{9}\right)$

$$
\tilde{\omega}_{\alpha}^{0}=0, \quad \tilde{\omega}_{0}^{\alpha}=0
$$

i rimanenti coefficienti $\tilde{\omega}_{\alpha}^{\beta}, \tilde{\omega}_{0}^{0}$ caratterizzano le connessioni ridotte $\nabla_{\sigma}$ e $\nabla_{\theta}$, il cui carattere metrico è espresso in $s$, se questa è $g$-ortonormale, dalle condizioni di antisimmetria $\left({ }^{10}\right)$

$$
\tilde{\omega}_{\beta}^{\alpha}+\tilde{\omega}_{\alpha}^{\beta}=0, \quad \tilde{\omega}_{0}^{0}=0
$$

Sussiste pertanto le seguente proposizione:

(7) Cf. [15], p. 212.

${ }^{(8)}$ Gli indici latini variano da 0 a 3 , quelli greci da 1 a 3.

(9) Cf. [15], p 216.

$\left({ }^{10}\right)$ Cf. [18], p. 118. 
1-3 Propostzione. - La connessione spazio-temporale $\nabla$ ammette, in ogni sezione $g$-ortonormale di $B(\Pi)$, coefficienti verificanti le condizioni $(1.10,11)$.

d) Sia $S=(I I, g, \nabla)$ un sistema spazio-temporale in $M$.

1-4 Defrnizione. - $S$ è un sistema spazio-temporale piatto se $\nabla$ è una connessione globalmente piatta in $M\left({ }^{11}\right)$.

La connessione globalmente piatta $\nabla$ stabilisce tra le fibre di $T M$ un isomorfismo canonico che consente di identificarle tutte con un unico spazio vettoriale $\boldsymbol{M}$. Nel suddetto isomorfismo si corrispondono isometricamente, stante (S3), sia le fibre di $\sigma$ che quelle di $\theta$. Di conseguenza, stante $(S 1)$ e $(S 2)$, il sistema piatto $S$ risulta caratterizzato nello spazio affine $(\boldsymbol{M}, \boldsymbol{M})$ come segue:

(i) La struttura $\Pi=(\sigma, \theta)$ risulta costituita da una coppia di sottospazi vettoriali supplementari di $\boldsymbol{M}(\operatorname{con} \theta$ direzione orientata). Questi definiscono in $M$ due relazioni di equivalenza (genidentità e simultaneità globali), caratterizzate da quozienti $\Sigma$ e $\Theta$ (spazio e tempo globali) costituiti dai sottospazi affini di direzione $\theta$ e $\sigma$ rispettivamente e muniti di naturali strutture affini $(\Sigma, \sigma),(\Theta, \theta)$ che rendono un isomorfismo la proiezione canonica di $M$ nel prodotto cartesiano $\Sigma \times \Theta$.

(ii) La metrica spazio-temporale $g=\left(g_{\sigma}, g_{\theta}\right)$ risulta costituita da una coppia di metriche strettamente euclidee (di opposte segnature) in $(\Sigma, \sigma)$ e $(\Theta, \theta)$, cui corrispondono in $M$ intervalli spaziale e temporale globali.

(iii) La connessione spazio-temporale $\nabla=\left(\nabla_{\sigma}, \nabla_{\theta}\right)$ risulta costituita dalla coppia delle connessioni piatte di $(\Sigma, \sigma)$ e $(\Theta, \theta)$, cui corrispondono le derivazioni ordinarie in $\sigma$ e $\theta$.

\section{2. - Classe canonica di sistemi spazio-temporali.}

\subsection{Definizione.}

Il carattere relativo (aI sistema di riferimento) delle nozioni di spazio e tempo, modellate in $M$ mediante la struttura di sistema spazio-temporale, induce a considerare in $M$ classi di sistemi siffatti.

Precisamente, ad ogni collezione di sistemi di riferimento corrisponderà in $M$ una classe canonica $O$ di sistemi spazio-temporali, definita come segue:

2-1 Definizione. - $C$ è una classe canonica di sistemi spazio-temporali in $\boldsymbol{M}$ se è

$$
C=\left\{S, S^{\prime}, \ldots\right\}
$$

(11) $\nabla$ è cioè caratterizzata, in coordinate globali su $M$, da coefficienti identicamente nulli. 
denotando $\left\{S, S^{\prime}, \ldots\right\}$ un insieme costituito da sistemi spazio-temporali in $M$ verificanti i seguenti assiomi:

(C1) Per ogni $S, S^{\prime} \in C$, ed in ogni punto di $M$, vale la disequazione $\left(^{12}\right)$

$$
g\left(\gamma, \gamma^{\prime}\right)<0
$$

(C2) Per ogni $S, S^{\prime} \in C$, ed in ogni punto di $M$, valgono le implicazioni $\left({ }^{13}\right)$

$$
\begin{aligned}
& \theta=\theta^{\prime} \Rightarrow \tau=\tau^{\prime} \\
& \sigma=\sigma^{\prime} \Rightarrow h=h^{\prime}
\end{aligned}
$$

e inoltre

$$
\sigma=\sigma^{\prime} \Rightarrow \nabla_{\sigma}=\nabla_{\sigma^{\prime}}^{\prime}
$$

\subsection{Proprietà.}

a) L'assioma ( $C 1)$ impone a $C$ una condizione di ammissibilità fisica.

Precisamente, siano $T_{o}$ la classe dei vettori del genere tempo (costituita dai vettori non nulli appartenenti alle distribuzioni temporali di $C$ ) ed $S_{O}$ la classe dei vettori del genere spazio (costituita dai vettori non nulli appartenenti alle distribuzioni spaziali di $O)$. $\mathrm{Da}(C 1)$ segue che dette classi sono disgiunte,

$$
T_{\mathrm{C}} \cap \boldsymbol{S}_{C}=\emptyset
$$

Inoltre ciascun sistema $S \in C$ realizza, mediante le sottoclassi

$$
\begin{aligned}
& T_{o}^{-}=\left\{u \in T_{o}: g(\gamma, u)>0\right\} \\
& T_{c}^{+}=\left\{u \in T_{c}: g(\gamma, u)<0\right\},
\end{aligned}
$$

una partizione passato-futuro $P_{S}$ di $T_{C}$ che, stante $(C 1)$, risulta invariante al variare di $S$ in $O$. Infatti, indicata con $u^{\prime}$ la componente del generico vettore $u \in T_{\sigma}$ lungo il generatore $\gamma^{\prime}$ della distribuzione temporale $\theta^{\prime}$ che lo contiene, per ogni $S \in C$ sussiste, stante (2.1), l'equivalenza.

$$
g(\gamma, u) \gtrless 0 \Leftrightarrow u^{\prime} \lessgtr 0
$$

(12) Per $S^{\prime}$ si adotteranno, munite di apici, le medesime notazioni introdotte per $S$.

$\left({ }^{13}\right)$ In corrispondenza al generico sistema spazio-temporale $\mathcal{S},(\tau, h)$ denoterà la coppia caratterizzata da $(1.7,8)$. 
il cui secondo membro è indipendente da $S$; ciò prova appunto la preannunciata condizione di invarianza

$$
P_{s}=P_{s^{\prime}} \quad\left(\forall S, S^{\prime} \in C\right)
$$

Inversamente, ove sussistano per ipotesi le condizioni $(2.5,6)$, risulta senz'altro verificato l'assioma ( $C 1)$. In tal caso infatti, per ogni $S, S^{\prime} \in C$ ed in ogni punto di $M$ non possono essere verificate nè la condizione $g\left(\gamma, \gamma^{\prime}\right)=0$ (che comporterebbe, in contrasto con (2.5), la relazione assurda $\left.\gamma^{\prime} \in T_{0} \cap S_{c}\right)$, nè la condizione $g\left(\gamma, \gamma^{\prime}\right)>0$ (che, coesistendo con $g^{\prime}\left(\gamma^{\prime}, \gamma^{\prime}\right)=-1$, comporterebbe, stante (2.6), la relazione assurda $\left.\gamma^{\prime} \in T_{c}^{-} \cap T_{c}^{+}\right)$; rimane quindi verificata la condizione (2.1) che prova l'asserto.

Sussiste pertanto la seguente proposizione:

2-2 Proposizione. - L'assioma $(O 1)$ è completamente equivalente alle condizioni $(2.5,6)$.

Le condizioni $(2.5,6)$, riguardando i vettori del genere tempo come traiettorie d'universo locali di particelle $\left({ }^{14}\right)$, hanno il seguente significato fisico: la condizione (2.5) è conforme all'assunzione che, rispetto a ciascun sistema di riferimento, ogni particella abbia velocità finita; la condizione (2.6) è conforme all'assunzione che sia invariante l'ordine temporale degli eventi causalmente connettibili.

b) L'assioma $(O 2)$ riduce $O$ ad una classe di sistemi spazio-temporali fisicamente distinti.

Precisamente, le condizioni $(2.2,3)$ richiedono che su ciascuna fibra delle distribuzioni spaziali e temporali di $C$ la struttura metrica, che è a priori determinata solo a meno di isomorfismi, sia univocamente fissata (la (2.2) richiede anche che a ciascuna fibra temporale risulti univocamente associato un ordinamento temporale locale in $M$ ).

Analogamente (derivando da (2.2) l'implicazione $\theta=\theta^{\prime} \Rightarrow \nabla_{\theta}=\nabla_{\theta^{\prime}}^{\prime}$ ) le condizioni $(2.2,4)$ richiedono che su ciascuna distribuzione spaziale e temporale di $C$ anche la connessione metrica sia univocamente fissata.

Se ne deduce, tenendo conto anche di (2.1), la seguente proposizione:

2-3 Proposizione. - I sistemi spazio-temporali $S, S^{\prime} \ldots$ costituenti una classe canonica hanno supporti fisici distinti, ovvero

$$
S \neq \mathbb{S}^{\prime} \Leftrightarrow \Sigma \neq \Sigma^{\prime}
$$

c) Si assuma ora che la classe canonica $C$ corrisponda alla totalità dei sistemi di riferimento.

In tal caso, $T_{C}$ dovrà essere riguardata come la totalità delle traiettorie d'universo locali di particelle; in particolare, ogni distribuzione $\theta$ del genere tempo (generata

$\left({ }^{14}\right)$ Cf. il comma a) del n. 1.2. 
cioè da sezioni di $T_{C}$ ) rappresenterà un continuo che, stante l'ipotesi dianzi assunta su $C$, dovrà necessariamente costituire il supporto fisico di un sistema $S \in C$.

Pertanto, nel caso in esame, $C$ deve possedere il seguente requisito di $T$-massimalità:

2-4 Definizione. - $O$ è una classe canonica $T$-massimale se, per ogni distribuzione $\theta$ del genere tempo, esiste un sistema $S \in C$ che ha $\theta$ come sua distribuzione temporale.

d) Sia $C$ una classe canonica di sistemi spazio-temporali in $M$.

2-5 Definizione. $-C$ è una classe canonica piatta se è costituita da sistemi piatti aventi la medesima connessione spazio-temporale.

Da un punto di vista geometrico, $C$ individua univocamente in $M$ una struttura di spazio affine $(M, \boldsymbol{M})$ recante una classe privilegiata di sistemi caratterizzati dai requisiti (i), (ii), (iii) di cui al $n .1 .2 d$ ).

Da un punto di vista cinematico, $C$ risulta costituita da sistemi reciprocamente animati di moto traslatorio uniforme. Basta infatti osservare che, indicato con $v=-\mathfrak{T}_{\mathrm{o}}\left(\gamma^{\prime}\right) / g\left(\gamma, \gamma^{\prime}\right)$ il campo delle velocità relative di due sistemi spazio-temporali $S$ ed $S^{\prime}$, dalla condizione $\nabla=\nabla^{\prime}$, verificata in $C$, si deduce la condizione $\nabla v=0$, che caratterizza appunto un moto traslatorio uniforme.

\section{3. - Modello canonico di spazio-tempo.}

\subsection{Definizione.}

In tutte le attuali teorie fisiche il modello matematico fondamentale d'universo $M$ si considera altresi munito di una connessione fondamentale $D$, necessaria ai fini della formulazione differenziale generalmente covariante delle teorie.

Introducendo in $M$ una tal connessione insieme ad una classe canonica di sistemi spazio-temporali, si perviene ad un modello canonico di spazio-tempo $K$, definito come segue:

3-1 Definizione. - $K$ è un modello eanonico di spazio-tempo se è

$$
K=(M, C, D),
$$

denotando $(M, C, D)$ un insieme costituito da una 4 -varietà $M$, una classe canonica $T$-massimale $C$ in $M$ ed una connessione affine $D$ in $M$, verificanti i seguenti assiomi:

(K1) Per ogni $S \in C$ valgono le relazioni

$$
\nabla_{\sigma}=\mathfrak{T}_{\sigma}(D), \quad \nabla_{\theta}=\mathfrak{T}_{\theta}(D) .
$$

(K2) Le classi $T_{0}$ ed $S_{O}$ sono $D$-invarianti $\left({ }^{15}\right)$.

${ }^{(15)}$ Il trasporto parallelo definito da $D$ in $T M$ trasforma vettori di $T_{o}$ (risp. $S_{\sigma}$ ) in vettori di $T_{c}$ (risp. $S_{\sigma}$ ).

Risultano poi separatamente $D$-invarianti le sottoclassi $T_{\sigma}^{-}, T_{\sigma}^{+}$. 


\subsection{Proprietà.}

a) L'assioma $(K 1)$ determina univocamente, per ogni $S \in C$, il criterio di scelta (che è a priori del tutto arbitrario) della relativa connessione spazio-temporale $\nabla$.

Precisamente, stante (3.1), $\nabla$ definisce, lungo la generica linea $l$ di $M$, un trasporto parallelo $\left(e_{i}\right)$ in $B(\Pi)$ che si identifica con il trasporto generalizzato di Fermi-Walker, sia della terna spaziale $\left(e_{\alpha}\right)\left({ }^{16}\right)$ e sia dell'asse temporale $e_{0}$, ivi definito da $D$ mediante le relazioni di parallelismo naturale

$$
\mathfrak{T}_{\sigma}\left(e_{\alpha}^{\prime}\right)=e_{\alpha}, \quad \mathfrak{T}_{\theta}\left(e_{0}^{\prime}\right)=e_{0},
$$

in cui la base $\left(e_{i}^{\prime}\right)$ è da intendersi definita dai differenziali, valutati lungo $l, D e_{i}=$ $=e_{i}^{\prime}-e_{i}$.

Dall'assioma $(K 1)$ si deduce la seguente proposizione:

3-2 Proposizione. - In un modello canonico $K$ sussistono, in corrispondenza a ciascun sistema $S \in C$, le equazioni di struttura

$$
\mathfrak{T}_{\sigma \sigma}(D g)=0, \quad \mathfrak{T}_{\theta \theta}(D g)=0
$$

o equivalentemente

$$
\mathfrak{T}_{\sigma \sigma}(D h)=0, \quad \mathfrak{T}_{\theta}(D \tau)=0 .
$$

Indicati infatti con $\omega_{j}^{i} i$ coefficienti di $D$ in una sezione $s$ di $B(\Pi)$, le condizioni (3.1) sono espresse da

$$
\tilde{\omega}_{\alpha}^{\beta}=\omega_{\alpha}^{\beta}, \quad \tilde{\omega}_{0}^{0}=\omega_{0}^{0} .
$$

Se poi $s$ è g-ortonormale, da (1.11) e (3.4) si deducono le equazioni

$$
\omega_{\alpha}^{\beta}+\omega_{\beta}^{\alpha}=0, \quad \omega_{0}^{0}=0
$$

che, in termini intrinseci, si scrivono nella forma (3.2) o (3.3).

b) L'assioma ( $K 2)$ esprime un naturale requisito di compatibilità fra le strutture componenti $C$ e $D$ imposte alla varietà di base $M$ dalla struttura composta $K$.

Da tale requisito consegue che:

3-3 Proposizione. - In un modello canonico $K$ l'insieme delle geodetiche della connessione fondamentale $D$ risulta ripartito nelle seguenti classi: geodetiche del

(16) Cf. [14], pp. 723 e seg. 
genere tempo, ovunque tangenti a vettori di $T_{c}$; geodetiche del genere spazio, ovunque tangenti a vettori di $S_{C}$; infine geodetiche ovunque tangenti a vettori di $L_{C}=T M-$ $-\left(T_{C} \cup S_{C}\right)$.

La suddetta ripartizione consente di individuare nell'insieme delle geodetiche di $D$ classi di traiettorie d'universo fisicamente privilegiate. In particolare, si indicherà con $C^{\circ}$ la sottoclasse privilegiata di $C$ costituita dai sistemi spazio-temporali a supporto geodetico, cioè dai sistemi $S \in C$ il cui supporto fisico $\Sigma$ è una congruenza di geodetiche, del genere tempo, di $D$.

c) Sia $K=(M, C, D)$ un modello canonico di spazio-tempo.

3-4 Defintzionf. - $K$ è un modello canonico piatto di spazio-tempo se

(i) $D$ è una connessione globalmente piatta in $M$;

(ii) per ogni $S \in C$, vale l'implicazione $D \theta$-riducibile $\Rightarrow D \sigma$-riducibile.

Stante (i), l'insieme dei campi vettoriali $D$-uniformi su $M$ è senz'altro non vuoto; in particolare risulta non vuoto, stante $(K 2)$, il sottoinsieme costituito dai campi del genere tempo, ciascuno dei quali genera, per la $T$-massimalità di $C$, il supporto, evidentemente geodetico, di un sistema $S \in C$. Risulta pertanto provato che:

3-5 Proposizione. - La classe $\stackrel{C}{C}$ dei sistemi a supporto geodetico è non vuota.

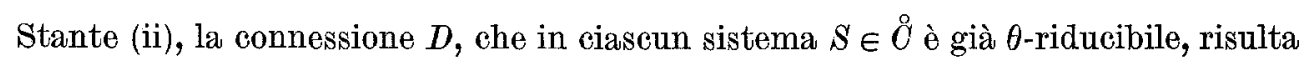
ivi altresì $\sigma$-riducibile; dette condizioni di riducibilità, unite alle condizioni (3.1), identificano $D$ con la connessione spazio-temporale $\nabla$ relativa ad $S$,

$$
D=\nabla \text {. }
$$

Ne segue che:

3-6 Proposizione. - $\stackrel{C}{C}$ è una classe canonica piatta, con connessione spaziotemporale $D$.

Valgono dunque per $\stackrel{C}{C}$ le considerazioni, geometriche e cinematiche, esposte al n. $2.2 d)$.

Dal punto di vista geometrico va peraltro precisato che la classe $\stackrel{C}{C}$ è completamente caratterizzata in $C$ dalla condizione

$$
D_{\gamma}=0
$$

Infatti, ogni sistema $S \in C$ verificante (3.7) ha un supporto evidentemente geodetico ed appartiene quindi a $\mathscr{C}$; viceversa, ogni sistema $S \in \stackrel{C}{C}$ verifica, stante 3-6, la condizione (3.6), da cui, tenendo conto di $(1.10)_{2}$ e $(1.11)_{2}$, segue la (3.7).

Rimane dunque provato che: 
3-7 Proposizione. - Appartengono a $\stackrel{C}{C}$ tutti e soli i sistemi $S \in C$ verificanti la condizione (3.7).

Dal punto di vista cinematico va inoltre precisato che $\stackrel{C}{C}$ è una classe completa di sistemi reciprocamente animati di moto traslatorio uniforme.

Infatti, la condizione $D v=0$, che caratterizza i moti traslatori uniformi di sistemi $S^{\prime} \in C$ rispetto ad un prefissato sistema $S \in \mathscr{C}$, risulta equivalente alla condizione $D \gamma^{\prime}=0$, che caratterizza, stante 3-7, l'appartenenza di $S^{\prime}$ a $\stackrel{\circ}{\text {. }}$

Rimane dunque provato che:

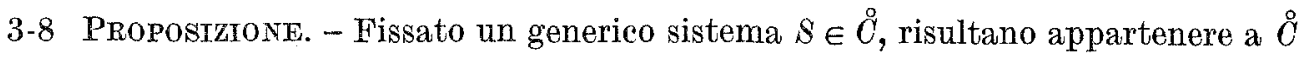
tutti e soli i sistemi $S^{\prime} \in O$ animati di moto traslatorio uniforme rispetto ad 8 .

\section{4. - Modello einsteiniano di spazio-tempo.}

\subsection{Definizione.}

Si assuma che in ogni sistema di riferimento un agente fisico $L$ (la luce) si propaghi isotropicamente con velocità $c=1$.

Indicata con $O$ una classe canonica $T$-massimale, rappresentativa della totalità dei sistemi di riferimento, si consideri, in corrispondenza a ciascun sistema $S \in C$, la struttura conforme $\operatorname{Ker}(g)\left({ }^{17}\right)$ definita in $M$ dalla relativa metrica spazio-temporale $g$.

Il principio di isotropia ottica dianzi enunciato si formula in $M$ richiedendo l'invarianza della suddetta struttura conforme al variare di $S$ in $C$, ovvero, per ogni $S$, $S^{\prime} \in C$,

$$
\operatorname{Ker}(g)=\operatorname{Ker}\left(g^{\prime}\right) \text {. }
$$

Da detta condizione di invarianza segue la relazione

$$
g=f g^{\prime}
$$

con $f$ funzione differenziabile su $M$ alla quale un principio di reciprocità impone di soddisfare anche la relazione

$$
g^{\prime}=f g
$$

che, insieme alla precedente, rende per essa possibili solo le determinazioni

$$
f=1, \quad f=-1
$$

(17) Si ̀̀ posto $\operatorname{Ker}(g)=\{u \in T M: g(u, u)=0\}$. 
di queste solo la prima è compatibile con l'assioma $(C 1)$, sicchè, per ogni $S, S^{\prime} \in C$, risulta

$$
g=g^{\prime} .
$$

Si è pertanto indotti ad assumere che la classe $C$ sia precisamente una classe cano . nica massimale di sistemi spazio-temporali in $M$ aventi la medesima metrica spaziotemporale. Denominata classe einsteiniana una siffatta classe canonica, si perviene per tal via ad un modello einsteiniano di spazio-tempo $E$, definito come segue:

4-1 Definizione. - $E$ è un modello einsteiniano di spazio-tempo se è

$$
E=(M, C, D),
$$

denotando $(M, O, D)$ un modello canonico di spazio-tempo verificante l'assioma $(E) C$ è una classe einsteiniana.

\subsection{Proprietà.}

a) Sia $E=(M, C, D)$ un modello einsteiniano di spazio-tempo.

I sistemi $S \in C$ definiscono in $M$ (stante l'assioma $(E)$ ) una metrica spazio-temporale assoluta $g$, con $\operatorname{Ker}(g)$ univocamente orientato (stante l'assioma (C1)) dai relativi generatori $\gamma\left({ }^{18}\right)$.

Inoltre la connessione fondamentale $D$ soddisfa l'equazione di struttura

$$
D g=0 .
$$

Infatti, in corrispondenza a ciascun sistema $S \in C$ risultano soddisfatte (stante 3-2) le equazioni di struttura (3.2); si perviene allora alla (4.1) mostrando che in $S$ risultano soddisfatte anche le equazioni

$$
\mathfrak{T}_{\sigma \theta}(D g)=0 \text {. }
$$

A tale scopo basta osservare che l'equazione del trasporto parallelo $D u=0$, lungo la generica linea di $M$, ammette come soluzioni (stante $(K 2)$ ) anche sezioni di $L_{c}$; per ognuna di tali soluzioni, in una sezione di $B(\Pi)$ si ha

$$
\left(D g_{x 0}\right) u^{\alpha} u^{0}=0
$$

${ }^{(18)}$ Le classi $T_{c}, L_{c}, S_{c}$ vengono a identificarsi rispettivamente con l'interno, la frontiera, l'esterno dei coni nulli costituenti le fibre di Ker $(g)$ e la partizione invariante $\left(T_{\sigma}^{-}, T_{\sigma}^{+}\right)$ di $T_{c}$ si identifica con quella ivi determinata dall'orientamento di $\operatorname{Ker}(g)$.

24 - Annali di Matematica 
da cui, potendo assegnare valori iniziali arbitrari ad $u^{\alpha}$, segue

$$
D g_{\alpha 0}=0
$$

ovvero l'asserto.

Sussiste pertanto la seguente proposizione:

4-2 Proposizione. - Passando da una classe einsteiniana $C$ alla corrispondente metrica spazio-temporale assoluta $g$, si viene a definire un'applicazione

$$
\Psi: E \rightarrow E^{\prime}
$$

che ad ogni modello einsteiniano di spazio-tempo

$$
E=(M, C, D)
$$

associa una struttura geometrica

$$
E^{\prime}=(M, g, D)
$$

costituita da una 4-varietà $M$ recante una metrica lorentziana $g$ con Ker $(g)$ orientato ed una connessione affine $D$ verificante (4.1).

Sia ora $E^{\prime}=(M, g, D)$ una struttura geometrica del tipo (4.3).

Dagli assiomi della teoria si deduce che un modello einsteiniano $E$ verificante la condizione

$$
\Psi(E)=E^{\prime}
$$

se esiste, è univocamente determinato ed è dato precisamente da $E=(M, C, D)$, dove $C$ denota la classe di tutti $\theta$ soli i sistemi $S=(\Pi, g, \nabla)$ così costituiti:

(i) $I I$ consiste di una distribuzione 1 -dimensionale $\theta$ interna ed equiorientata a $\operatorname{Ker}(g)\left({ }^{19}\right)$;

(ii) $g \in E^{\prime}\left({ }^{20}\right)$;

(iii) $\nabla$ è data, in funzione di $D \in E^{\prime}$, da (1.3), (3.1) ( $\left.{ }^{21}\right)$.

(19) Per l'esistenza di una siffatta distribuzione, cf. [19], p. 531.

(20) La l-forma temporale e la metrica spaziale dedotte da $g$, in corrispondenza a $\Pi$, mediante $(1.7,8)$ si identificano con il tempo standard e la metrica spaziale standard introdotti in [8].

(21) Le connessioni verificanti, in corrispondenza a $I I$, la condizione (1.3) si identificano con le connessioni adattate introdotte in [24]. In particolare, la connessione (1.3), (3.1) si identifica con la connessione vincolata introdotta in [25]. 
D'altra parte, con tale determinazione della classe $C$ (senz'altro non vuota), risultano soddisfatti, stante (4.1), tutti gli assiomi che garantiscono che $E$ sia effettivamente un modello einsteiniano di spazio-tempo.

Rimane pertanto provato che:

4-3 Proposizione. - L'applicazione $\Psi$ è biiettiva.

Le proposizioni 4-2 e 4-3 forniscono una caratterizzazione del modello einsteiniano di spazio-tempo (4.2) in termini della ben nota struttura geometrica (4.3), costituente lo spazio-tempo di Einstein-Cartan $\left({ }^{22}\right)$ del quale la formulazione (4.2) fornisce la completa analisi cronogeometrica.

b) Sia $E=(M, C, D)$ un modello einsteiniano di spazio-tempo con connessione fondamentale $D$ piatta.

In tal caso $C$ definisce univocamente in $M$, stante (4.1), una metrica $g$ minkowskiana e $\Psi(E)$ è lo spazio-tempo di Minkowski $(M, g)$.

Sussistono le seguenti proposizioni:

4-4 Propostzione. - $E$ è un modello canonico piatto.

Basta infatti osservare che, stante (4.1), per ogni $S \in C$ sono soddisfatte, nella generica sezione $g$-ortonormale $s$ di $B(\Pi)$, le equazioni

$$
D g_{\alpha 0}=\omega_{\alpha}^{0}-\omega_{0}^{\alpha}=0 ;
$$

poiché d'altra parte le condizioni di $\theta$ e $\sigma$-riducibilità di $D$ sono espresse in $s$ dalle equazioni

$$
\omega_{0}^{\alpha}=0, \quad \omega_{\alpha}^{0}=0,
$$

risulta verificata anche la condizione (ii) della definizione 3-4.

4-5 Propostzione. - $\stackrel{C}{C}$ si identifica con la classe minkowskiana costituita dai sistemi spazio-temporali piatti di metrica $g$.

Basta infatti osservare che, stante (4.1), $C$ esaurisce l'intera classe dei sistemi spaziotemporali piatti appartenenti a $C$, la quale, per la costituzione di $C$, coincide appunto con la suddetta classe minkowskiana.

\section{5. - Modello newtoniano di spazio-tempo.}

\subsection{Definizione.}

Si assuma ora che l'agente fisico $L$ (la luce) in ogni sistema di riferimento si propaghi isotropicamente con velocità $e=\infty$.

${ }^{(22)}$ Cf. [23]. 
Indicata con $O$ una classe canonica $T$-massimale, rappresentativa della totalità dei sistemi di riferimento, si consideri, in corrispondenza a ciascun sistema $S \in C$, la struttura conforme (degenere) $\operatorname{Ker}(\tau)$ definita in $M$ dalla relativa 1-forma temporale $\tau$.

Il limite newtoniano dianzi accolto si formula in $M$ richiedendo l'invarianza della suddetta struttura conforme al variare di $S$ in $C$, ovvero, per ogni $S, S^{\prime} \in C$,

$$
\operatorname{Ker}(\tau)=\operatorname{Ker}\left(\tau^{\prime}\right)
$$

Da detta condizione di invarianza segue la relazione

$$
\tau=f \tau^{\prime}
$$

con $f$ funzione differenziabile su $M$ alla quale un principio di reciprocità impone di soddisfare anche la relazione.

$$
\tau^{\prime}=f \tau,
$$

che, insieme alla precedente, rende per essa possibile solo le determinazioni

$$
f=1, \quad f=-1
$$

di queste solo la prima è compatibile con l'assioma (C1), sicchè, per ogni $S, S^{\prime} \in C$, risulta

$$
\tau=\tau^{\prime}
$$

da cui, stante (2.3), segue anche

$$
h=h^{\prime} .
$$

Si è pertanto indotti ad assumere che la classe $C$ sia precisamente una classe canonica massimale di sistemi spazio-temporali in $M$ aventi la medesima 1-forma temporale e la medesima metrica spaziale. Denominata classe newtoniana una siffatta classe canonica, si perviene per tal via ad un modello newtoniano di spazio-tempo $N$, definito come segue:

5-1 Definizione. - $N$ è un modello newtoniano di spazio-tempo se è

$$
N=(M, C, D)
$$

denotando $(M, C, D)$ un modello canonico di spazio-tempo verificante l'assioma $(N) C$ è una classe newtoniana. 


\subsection{Proprietà.}

a) Sia $N=(M, C, D)$ un modello newtoniano di spazio-tempo.

I sistemi $S \in C$ definiscono in $M$ (stante l'assioma $(N)$ ) una 1-forma temporale assoluta $\tau\left({ }^{23}\right)$ ed una metrica spaziale assoluta $h$.

Inoltre la connessione fondamentale $D$ soddisfa le equazioni $d i$ struttura

$$
D \tau=0, \quad D h=0 .
$$

Infatti, in corrispondenza a ciascun sistema $S \in C$ risultano soddisfatte (stante 3-2) le equazioni di struttura (3.3); si perviene allora alle (5.1) mostrando che in $S$ risultano soddisfatte anche le equazioni

$$
\int_{\sigma}(D \tau)=0
$$

A tale scopo basta osservare che l'equazione del trasporto parallelo $D u=0$, lungo la generica linea di $M$, ammette come soluzioni (stante $(K 2)$ ) anche sezioni di $S_{C}$; per ognuna di tali soluzioni, in una sezione di $B(\Pi)$ si ha

$$
\left(D \tau_{\alpha}\right) u^{\alpha}=0 \text {, }
$$

da cui, potendo assegnare valori iniziali arbitrari ad $u^{\alpha}$, segue

$$
D \tau_{\alpha}=0
$$

ovvero l'asserto $\left({ }^{24}\right)$.

Sussiste pertanto la seguente proposizione:

5-2 Proposizione. - Passando da una classe newtoniana $C$ alle corrispondenti metriche spaziale e temporale assolute $(\tau, h)$, si viene a definire un'applicazione

$$
\Phi: N \rightarrow N^{\prime}
$$

che ad ogni modello newtoniano di spazio-tempo

$$
N=(M, C, D)
$$

associa una struttura geometrica

$$
N^{\prime}=(M, \tau, h, D)
$$

${ }^{(23)}$ Le classi $T_{c}$ ed $S_{c}$ vengono a identificarsi rispettivamente con l'interno e la frontiera dei coni nulli (degeneranti in iperpiani) costituenti le fibre di Ker $(\tau)$ e la partizione invariante $\left(T_{\sigma}^{-}, T_{\sigma}^{+}\right)$di $T_{\sigma}$ si identifica con quella ivi determinata dal $\tau$-orientamento.

$\left({ }^{24}\right)$ Se $D$ è simmetrica, l'equazione $(5.1)_{1}$ assicura in particolare l'integrabilità della distribuzione $\operatorname{Ker}(\tau)$ (cf. [5], p. 447). 
costituita da una 4-varietà $M$ recante una 1-forma $\tau$ ovunque non nulla, una metrica fibrata $h$ in $\operatorname{Ker}(\tau)$ definita positiva ed una connessione affine $D$ verificante (5.1).

Sia ora $N^{\prime}=(M, \tau, h, D)$ una struttura geometrica del tipo (5.3).

Dagli assiomi della teoria si deduce che un modello newtoniano $N$ verificante la condizione

$$
\Phi(N)=N^{\prime},
$$

se esiste, è univocamente determinato ed è dato precisamente da $N=(M, C, D)$, dove $C$ denota la classe di tutti e soli i sistemi $S=(\Pi, g, \nabla)$ così costituiti:

(i) II consiste di una distribuzione 1-dimensionale $\theta \notin \operatorname{Ker}(\tau)\left({ }^{25}\right), \tau$-orientata, e da $\sigma=\operatorname{Ker}(\tau)$;

(ii) $g$ è data, in funzione di $\tau, h \in N^{\prime}$, da (1.6);

(iii) $\nabla$ è data, in funzione di $D \in N^{\prime}$, da (1.3), (3.1).

D'altra parte, con tale determinazione della classe $C$ (senz'altro non vuota), risultano soddisfatti, stante (5.1), tutti gli assiomi che garantiscono che $N$ sia effettivamente un modello newtoniano di spazio-tempo.

Rimane pertanto provato che:

\section{5-3 Proposizione. - L'applicazione $\Phi$ è biiettiva.}

Le proposizioni 5-2 e 5-3 forniscono una caratterizzazione del modello new toniano di spazio-tempo (5.2) in termini della ben nota struttura geometrica (5.3), costituente lo spazio-tempo di Newton-Cartan (analogo newtoniano dello spazio-tempo relativistico di Einstein-Cartan) del quale la formulazione (5.2) fornisce la completa analisi cronogeometrica.

b) Sia $N=(M, C, D)$ un modello newtoniano di spazio-tempo con connessione fondamentale piatta.

In tal caso $C$ definisce in $M$, stante (5.1), una metrica $(\tau, h)$ galileiana $\left({ }^{26}\right)$ e $\Phi(N)$ è lo spazio-tempo di Galitei $(M, \tau, h)$.

Poiché da (5.1) segue la riducibilità di $D$ in Ker $(\tau)$ e ricordando la costituzione di $C$, sussistono le seguenti proposizioni:

5-4 Proposiztone. - $N$ è un modello canonico piatto.

5-5 Proposizione. - 8 si identifica con la classe galileiana costituita dai sistemi spazio-temporali piatti di metrica $(\tau, h)$.

$\left({ }^{25}\right)$ Per l'esistenza di una siffatta distribuzione, cf. [5], p. 447.

${ }^{26}$ ) La metrica $(\tau, h)$ è cioè caratterizzata, in coordinate globali su $M$, da componenti costanti. 


\section{BIBLIOGRAFIA}

[1] A. Trudtman, Invariance of Lagrangian systems, in L. O'Raifeartaigh (ed.), General Relativity, Clarendon Press, Oxford (1972).

[2] A. Trautman, Sur la théorie newtonienne de la gravitation, Comp. Rend. Paris, 257, (1963), p. 617 .

[3] A. Tratuman, Comparison of Newtonian and relativistic theories of space-time, in B. Hoffman (ed.), Perspectives on Geometry and Relativity, Indiana University Press, Bloomington (1966).

[4] P. Havas, Four-dimensional formulations of Newtonian mechanics and their relation to the special and the general theory of relativity, Rev. Mod. Phys., 36 (1964), p. 938.

[5] H. P. Kunzte, Covariant Newtonian limit of Lorentz space-times, Gen. Rel. Grav., 7 (1976), p. 445.

[6] R. Penrose, The structure of space-time, in C. M. De Witt, J. A. Wheeler (ed.), Battelle Rencontres, Benjamin Ine. (1968).

[7] J. EnLers - F. A. E. PirAni - A. SCHILd, The geometry of free fall and light propagation, in L. O'Raifeartaigh (ed.), General Relativity, Clarendon Press, Oxford (1972).

[8] C. CATTANE0, General relativity: relative standard mass, momentum, energy and gravitational field in a general system of reference, Il Nuovo Cimento, 10 (1958), p. 318.

[9] C. CatTaxeo, Introduzione alla teoria einsteiniana della gravitazione, Veschi, Roma (1961).

[10] C. CaTtaneo, Sur la loi relative du mouvement d'une 'particule d'épreuve gravitant libre ment, Compt. Rend. Paris, 256 (1963), p. 3974.

[11] F. EASTBRooK - H. WAHLQUIST, Dyadic analysis of space-time congruences, J. Math. Phys., 5 (1964), p. 1629.

[12] C. MøLler, The Theory of Relativity, Clarendon Press, Oxford (1972).

[13] C. Harrison, On the structure of space-time, in P. Suppes (ed.), Space, Time and Geometry, Reidel Publ. Co. (1973).

[14] R. GRassini, Estensione del trasporto misto di Fermi ad un modello generale di spaziotempo, Atti Acc. Naz. Lincei, Rend. Cl. Sci. Fis. Mat. Nat., 56 (1974), p. 720.

[15] R. Grassint, Riferimenti fisici e relative connessioni spaziali in un modello matematico generale di spazio-tempo, Rend. Acc. Sci. Fis. Mat. Napoli, 41 (1974), p. 209.

[16] R. Grassini, Misura ed evoluzione di grandezze fisiche relative in un modello matematico generale di spazio-tempo, Boll. U.M.I., 11 (1975), p. 507.

[17] A. Trautman, Foundations and current problems of general relativity, in S. Deser K. Ford (ed.), Lectures on General Relativity, Brandeis Summer Institute in Theoretical Physics, vol. 1, Prentice-Hall, Englewood Cliffs (1965).

[18] S. KoваYAshi - K. Nomizu, Foundation of Differential Geometry, vol. 1, Interscience Publ. (1963).

[19] G. S. Whiston, Topics on space-time geometry, Gen. Rel. Grav., 5 (1974), p. 526.

[20] A. Trautman, Fibre bundles associated with space-time, Rep. Math. Phys., 1 (1970), p. 29.

[21] P. SuPPEs, Introduction to Logie, Van Nostrand Co. (1967).

[22] J. D. Sneed, The Logical Structure of Mathematical Physics, Reidel Publ. (1971).

[23] A. Trautman, On the structure of Einstein-Cartan equations, Symp. Math., 12 (1973), p. 139.

[24] E. Massa, Space tensors in general relativity, Gen. Rel. Grav., 5 (1974), p. 555.

[25] I. Cattaneo Gasparint, Derivée covariant liée dans une $V_{n+1}$ riemannienne à structure presque produit, Compt. Rend. Paris, 256 (1963), p. 2089. 